\title{
Cluster persistence of two-dimensional soap froth
}

\author{
Wing Yim Tam and Kwok Yip Szeto \\ Department of Physics, The Hong Kong University of Science and Technology, Clear Water Bay, Kowloon, Hong Kong
}

(Received 30 August 2001; published 20 March 2002)

\begin{abstract}
We report on the persistence decay of cell clusters in dynamical simulation of two-dimensional soap-froth as a function of the cluster volume fraction. Both slow gas diffusion and fast dynamical topological processes between cells are incorporated in the simulation. While the simulation yields decay rate in good agreement with two-dimensional soap-froth experiment, it shows a faster decay in comparison to measurements and calculations of persistence in other curvature-driven systems. The fraction of individual cells that contain persistent area also decays due to significant cell movements, confirming that the neighbor swapping $T 1$ topological transition is important in froth coarsening.
\end{abstract}

DOI: 10.1103/PhysRevE.65.042601

PACS number(s): 82.70.Rr, 02.50.-r

Foams are three-dimensional, space-filling cellular structures analogous to other systems such as metal grains and biological tissues [1]. The simplest foam is made of soap bubbles where gas diffusion between bubbles drives the system to minimize the surface energy. As a result, the foam coarsens with the average bubble size increasing with time at the expense of the total number of bubbles in the foam. However, the study of three-dimensional foam had been hindered by the inability to acquire the internal structure of the foam [2] and it is only recently that detailed informations are obtained by different methods, like the magnetic resonance imaging [3], diffusing-wave spectroscopy [4], and optical tomography reconstruction [5]. These experimental studies, together with the recent theoretical developments [6], are leading to better understanding of the dynamics of soap foam. In contrast to three-dimensional foam, two-dimensional soapfroth had attracted much attention in the past decade because of its simplicity and relative ease in its experimental setup [7-10]. Again, driven by gas diffusion, it is found that cells grow (shrink) if their number of edges are larger (smaller) than six [11]. Furthermore, the system will evolve to a universal scaling state where the distribution of cell edges is time independent for almost all initial conditions [10,12]. The above static properties had been well studied experimentally and good agreements with theories and simulations had been reported [10,13-15]. Recently, much of the focus has shifted to the dynamics such as the studies of survivors $[16,17]$, first-passage exponent $[18,19]$, and cell movements [20]. It is now known that cell movement due to fast topological transitions caused by gas diffusion, specially the neighbor swapping (T1) process [21], plays an important role in the coarsening of soap-froth. This is in contrast to grain growth in polycrystallite systems where topological processes occur at about the same slow time scale as diffusion. To probe the coarsening dynamics, a new tool called cluster persistence, was introduced by Lee and Rutenberg to study the coarsening of soap-froth as well as noncellular structures [22]. We have applied the persistence to twodimensional soap-froth experiment and obtained a much faster decay of persistence than measurements and calculations of other systems, including the $Q$ state Potts model that is believed to resemble soap-froth for large $q$ [23]. The result suggests that soap froth is quite unique and needs further investigation.
Two-dimensional soap-froth has also been simulated using topological models [16] and direct dynamical simulation [24] using the method of Weaire and Kermode [25]. Direct dynamical simulation serves as a good test for the persistence. However, simulations by Weaire and Kermode (WK) [25] and later by Aref and Herdtle ( $\mathrm{AH})$ [26] were limited to small sample size and had not been compared to experiments. Recently, a much faster and larger dynamical simulation has been reported, which shows excellent agreement with soap froth [27]. In this paper, we report on the persistence of two-dimensional soap-froth simulated by using the dynamical model of Chae and Tabor $[27,28]$. The persistence shows faster decay rates for all cluster volume fractions in good agreement with the soap-froth experiment. Furthermore, large cell motions due to fast topological transitions are also observed in the simulation, explaining the fast decay rate.

The direct simulation has been reported recently in detail $[27,28]$ and is similar to that of WK [25] and $\mathrm{AH}[26]$. The simulation is based on a physical model where the movement of cell edges and vertices due to gas diffusion through the edges between neighboring cells is followed by finding the quasiequilibrium configuration such that the three edges meet at $120^{\circ}$ and the pressure difference across each edge is balanced by the tension acting on the edge after each "diffusion step." Furthermore, $T 1$ and $T 2$ topological transitions are implemented with a series of criteria to eliminate spurious behavior and enable tight control on the accuracy (see Refs. [27,28] for detailed implementation). Note that, unlike the simulations of WK [25] and AH [26], von Neumann's law is not built into the model but is well satisfied for every individual $n$-sided cell [27]. The model has been shown to reproduce quantitatively the evolution of two-dimensional soap froth $[27,28]$ and has also been applied to recent studies of ancestral cells (ancestors) that survive for long times [17] and to the evolution of a single defect in ideal twodimensional hexagonal soap-froth [20].

The simulation starts with 5000 cells obtained by using a Voronoi construction. Using periodic boundary conditions, the froth is allowed to evolve according to the above specified dynamics and stops at about 100 cells. Scaling state is obtained at about 3000 cells after a transient period. In the scaling state the mean cell area grows linearly with time and 

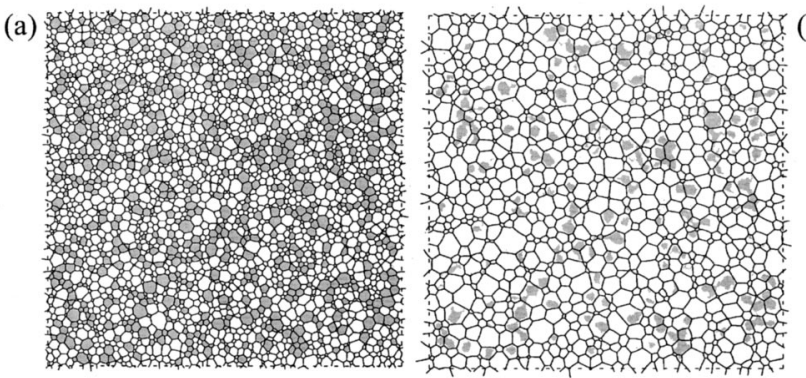

(c)
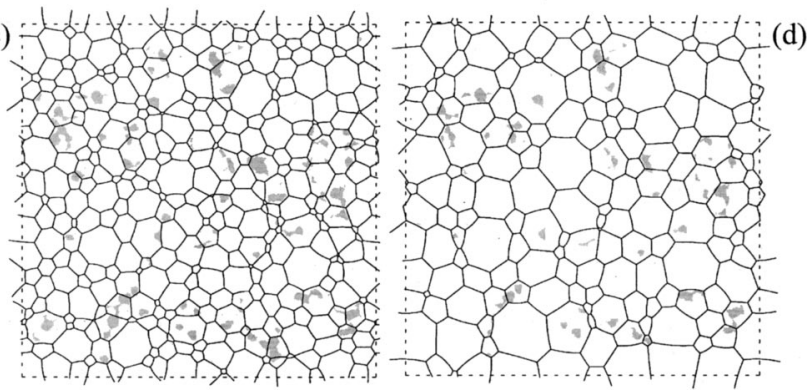

FIG. 1. Images of simulated froth (dark lines) taken at (a) 10, (b) 50, (c) 150, and (d) 300 (arbitrary time unit), with (a) 2887, (b) 865, (c) 304, and (d) 138 cells, respectively. Shaded areas show cluster persistence for initial volume fraction $\phi=0.31$ at different time.

the distribution of edges is stationary, which is in excellent agreement with the soap-froth experiment. Figure 1 shows the evolution of the froth (dark lines) in the scaling regime.

Following the previous definition for persistence, we define the pixels in a subset of cells $\mathcal{S}$ by a function $p_{\mathcal{S}}\left(j, t, t_{0}\right)=1$, where $j \in[1,1048,576]$ counts of the $M$ pixels, and for pixels outside of that subset of cells, $p_{\mathcal{S}}\left(j, t, t_{0}\right)$ $=0$. The cluster persistence of the set $\mathcal{S}$ is then given by

$$
P_{\mathcal{S}}\left(t, t_{0}\right)=\frac{1}{M} \sum_{j=1}^{M} \prod_{t^{\prime}=t_{0}}^{t} p_{\mathcal{S}}\left(j, t^{\prime}, t_{0}\right),
$$

where $0 \leqslant P_{\mathcal{S}}\left(t, t_{0}\right) \leqslant 1$. In other words, a pixel counts towards the persistence if it has always been present in any of the cells belonging to $\mathcal{S}$. With this definition, the traditional measure of persistence (or first-passage exponent) corresponds to the case where $\mathcal{S}$ consists of a single cell. More generally we can take arbitrary subsets of all of the $N\left(t_{0}\right)$ cells, where there are $2^{N\left(t_{0}\right)}$ possible subsets. We sample subsets $\mathcal{S}=\mathcal{S}(\phi)$ by randomly choosing $m \sim \phi N\left(t_{0}\right)$ cells at a time. Here $\phi=\overline{\sum_{j=1}^{m} A_{j}(t) / \sum_{j=1}^{N\left(t_{0}\right)} A_{j}\left(t_{0}\right)}$ (time averaged) is the cluster volume fraction. Figure 1 also shows the persistent area evolution (shaded areas) for initial cluster volume fraction $\phi=0.31$ with initial 2887 and final 138 cells. The resulting persistence decay is characterized by a power law

$$
P_{\mathcal{S}(\phi)}\left(t, t_{0}\right)=P_{\phi}(t) \sim t^{-\theta(\phi)} \sim\langle A\rangle^{-\theta^{\prime}(\phi)} .
$$

We expect $\theta^{\prime}=\theta$, since the average cell area in the froth $\langle A\rangle$ is asymptotically proportional to $t[10]$, but we measure $\theta^{\prime}$ to eliminate the dependence on the absolute time origin $t_{0}$ and to minimize transient effects $[17,29]$. We fit the persistent

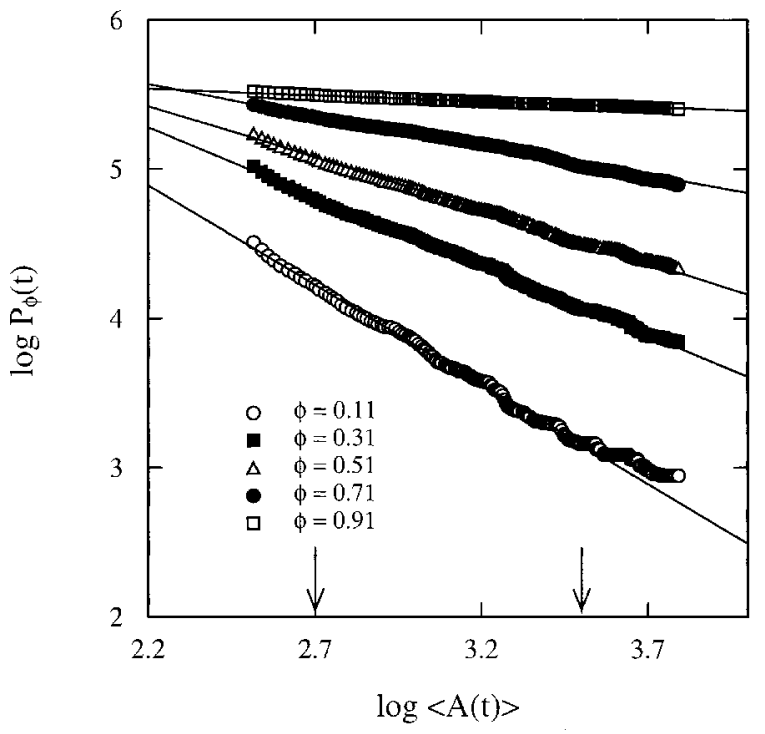

FIG. 2. Log-log [base 10] plot of cluster persistence (arbitrary unit) decay for various volume fractions $\phi$. Solid lines are linear fits to the data. The arrows indicate the fitting region used to extract all persistence exponents.

decay for individual subsets as shown in Fig. 2, and then average the resulting exponents-binning them with respect to $\phi(\mathcal{S})$. The averaged resulting exponents are plotted in Fig. 3 as a function of volume fraction. The errors shown are from the statistical variation of about 700 multiple-cell subsets out of the $2^{2887}$ possibilities for different volume fractions, and can be reduced with more sampling. The result is not sensitive to the fitting range and the choice of initial $t_{0}$ in

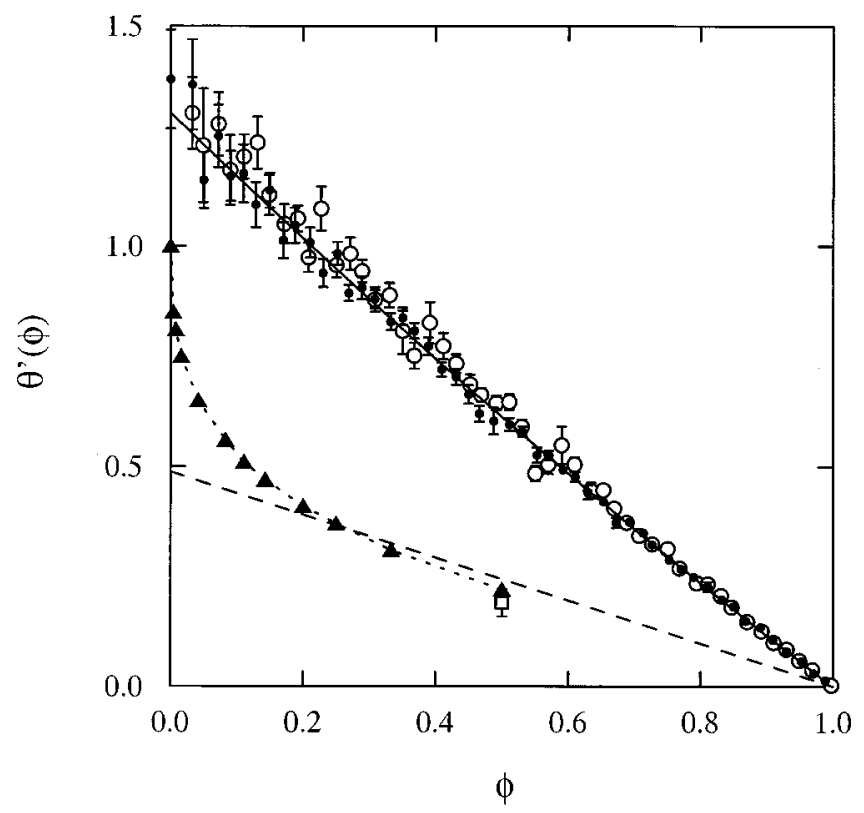

FIG. 3. Persistence decay exponent as a function of volume fraction for the direct simulation (open circles), the experimental soap froth (solid dots), Potts model with $\phi=1 / q$ (triangles), twisted nematics (open square), and droplet calculation (dashed line). The dotted curve is just a guide to the eye. 
the scaling regime. Also shown is the experimental result of soap-froth (solid dots) reported earlier [23]. It is clear that the agreement between the simulation and the experiment is very good for the entire range of volume fraction, with the simulation slightly higher than the experimental result. It is believed that better agreement can be obtained by fine tuning the details of the $T 1$ and $T 2$ topological processes in the simulation. For comparison, the data from previous studies of other systems with curvature-driven growth are also included. These include the persistence of the $Q$-state Potts model, with $\phi=1 / q$, simulated $[30,31]$ and plotted as triangles for $0<\phi \leqslant 1 / 2$. The Potts system, particularly when $q \rightarrow \infty$, has been proposed as soap-froth analogies [8]. The Potts data fits relatively well on a continuous curve (dotted line), interpolating smoothly between $q=2$ with a twophase, noncellular structure, $3<q<\infty$ with a cellular structure and some cell coalescence, and $q=\infty$ with a cellular structure and no cell coalescence [30]. Also shown (open square) is a previous persistence experiment on twisted nematic liquid crystals [32], an Ising model analog with $\phi=1 / 2$. Finally, an analytic result for a mean-field droplet model, valid for $\phi \approx 1$, describing nonconserved order parameter dynamics with a time-dependent applied field is plotted as a dashed line [22]. Overall, there is a good agreement between the Potts model, the experimental work on twisted nematics, and the droplet model. It is noted that neither the nematic nor the droplet systems have cellular structures, and the relatively good agreement between them and the Potts model indicates that persistence is not greatly affected by the cellular structure per se. However, the decay exponents of the soap-froth experiment and direct simulation are significantly larger than all these corresponding systems.

To understand the strong persistence decay in soap-froth relative to other curvature-driven systems, we find it more transparent to first look at the persistence of isolated cells. The persistence exponent obtained by extrapolation to $\phi$ $=0$, using a quadratic fit with powers of $(1-\phi)$, is 1.45 , which is slightly higher than 1.3 obtained from the experimental result [23]. However, it is significantly higher than the Potts model result of $\theta(0)=1$. Our result of $\theta$ being significantly greater than 1 implies either a continual erosion of the persistent cores in cells, a continual decrease in the fraction of cells with persistent cores, or both. Since the persistence of individual cells $\left(P_{0}^{*}\right)$ can be estimated from the product of the averaged persistent area per unit cell $\left(\left\langle A^{*}(t)\right\rangle\right)$ and the number of cells containing persistent area $\left(N^{*}\right)$, it would be instructive to study their time dependence. It is found that the average persistent area $\left\langle A^{*}(t) / A\left(t_{0}\right)\right\rangle$, where averages are restricted to the $N^{*}(t)$ cells that contain persistent areas at time $t$, reaches a steady value of about 0.3 for long times, which is in good agreement with the experimental result [23]. However, $N^{*}(t)$ is found to decay faster than $t^{-1}$ [note that $N(t) \sim t^{-1}$ ] and a log-log fit gives an exponent $-1.40 \pm 0.02$ that is consistent with the experimental value $(-1.27)$ [23]. Thus, for constant $\left\langle A^{*}(t) / A\left(t_{0}\right)\right\rangle$, $P_{0}^{*} \propto N^{*}$ accounting for the decay of single cells with decay rate consistent with the extrapolated values for the simulation and the experiment.

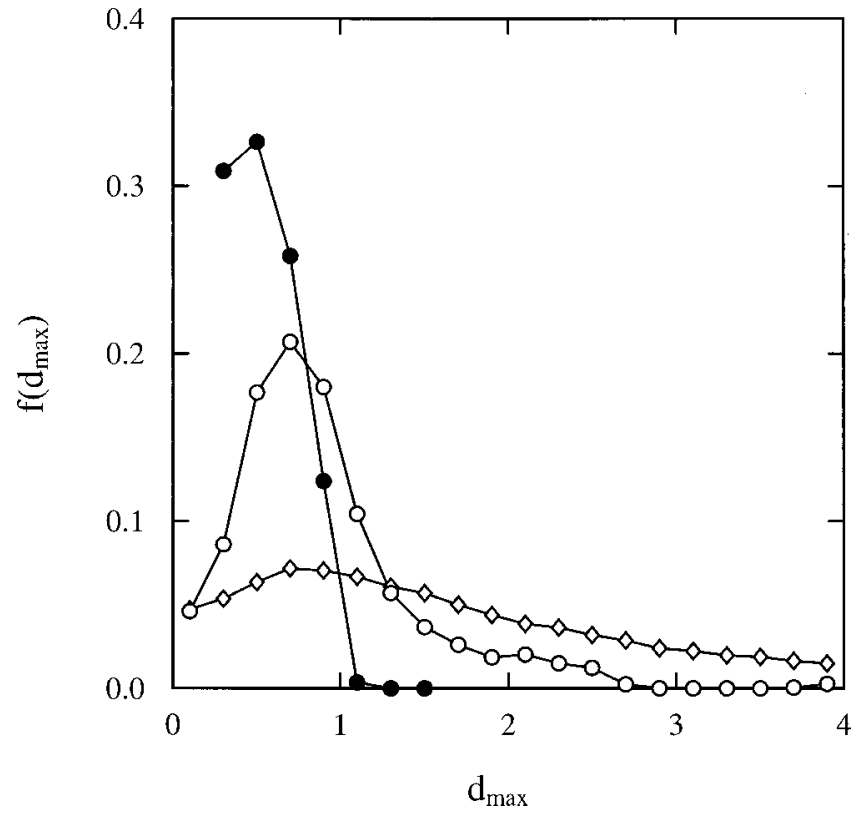

FIG. 4. The distribution of maximum normalized displacement $[d=|\mathbf{r}(t)-\mathbf{r}(0)| / \sqrt{A(t)}]$ for nonsurvivor (open diamond), survivor without persistent area (open circle), and survivor with persistent area (solid circle).

This power-law decay of $N^{*}(t) / N(t)$ implies significant movement of the center of mass of a cell, since persistence within a given surviving cell cannot be entirely lost without the cell moving off of its original center of mass. Cell motion on the scale of the cell size is therefore directly implicated in the strong persistence decay of soap-froth. This is supported by recent measurement of cell motion of two-dimensional soap-froth [20]. Figure 4 shows the distributions of maximum cell displacement obtained from the simulation for different types of cells. Cell motion is usually large (compared to cell size) for cells that do not survive to the latest time (nonsurvivor) and cells that do not contain persistent area, but it is small for cells containing persistent area. This is in good agreement with the experimental result [20]. It is clear from the simulation that large displacements are caused by the fast topological processes, especially the $T 1$ process that is often ignored in theoretical work $[13,14]$, and are responsible for the strong decay. This is consistent with results of direct simulation that show that the width of the distribution of cell edges $\mu_{2}$ is dependent on the specific implementation of $T 1$ process [28]. Furthermore, from our measurements of the $T 1$ processes that take place in real soap-froth [33], we can say that the $T 1$ in soap-froth evolution is probably the key process that distinguishes soap-froth from other systems such as $Q$-state Potts model, nematic, and droplet systems.

To conclude, we have used a dynamical model to simulate two-dimensional soap-froth to study cluster persistence. The persistence is found to decay faster than other curvaturedriven systems, especially the $Q$-state Potts Model that is believed to simulate the soap-froth for $q \rightarrow \infty$, for all volume fractions. The stronger decay is caused by cell motions triggered by fast topological processes. The result is in good agreement with the soap-froth experiment indicating that the 
dynamical simulation is a good model to study soap-froth evolution. Furthermore, it supports that the cluster persistence is an effective tool to probe the dynamics of coarsening systems. Lastly, although the current $Q$-state Potts Model does not agree with soap-froth, it could obtain better agreement if the topological processes in soap-froth are incorporated in the model.
We acknowledge valuable discussions with A. D. Rutenberg and B. P. Vollmayr-Lee. The code for the simulation is obtained from J. J. Chae and M. Tabor. The support from the Competitive Earmarked Research Grant No. HKUST 6123/ 98P of the Research Grants Council of Hong Kong is acknowledged.
[1] D. Weaire and N. Rivier, Contemp. Phys. 25, 59 (1984).

[2] E. Matzke, Am. J. Bot. 33, 58 (1946).

[3] C.P. Gonatas, J.S. Leigh, A.G. Yodh, J.A. Glazier, and B. Prause, Phys. Rev. Lett. 75, 573 (1995).

[4] A.D. Gopal and D.J. Durian, Phys. Rev. Lett. 75, 2610 (1995); J.C. Earnshaw and M. Wilson, J. Phys. II 6, 713 (1996); S. Cohen-Addad and R. Hohler, Phys. Rev. Lett. 86, 4700 (2001).

[5] C. Monnereau and M. Vignes-Adler, Phys. Rev. Lett. 80, 5228 (1998); C. Monnereau, B. Prunet-Foch, and M. Vignes-Adler, Phys. Rev. E 63, 061402 (2001).

[6] C. Sire, Phys. Rev. Lett. 72, 420 (1994); S. Hilgenfeldt, A.M. Kraynik, S.A. Koehler, and H.A. Stone, ibid. 86, 2685 (2001).

[7] J.A. Glazier, S.P. Gross, and J. Stavans, Phys. Rev. A 36, 306 (1987).

[8] J.A. Glazier, M.P. Anderson, and G.S. Grest, Phys. Mag. 62, 6115 (1990).

[9] E.A. Holm, J.A. Glazier, D.J. Srolovitz, and G.S. Grest, Phys. Rev. A 43, 2662 (1991).

[10] J. Stavans, Rep. Prog. Phys. 56, 733 (1993).

[11] J. von Neumann, Metal Interfaces (American Society of Metals, Cleveland, 1952), p.108.

[12] K.Y. Szeto and W.Y. Tam, Physica A 221, 256 (1995).

[13] H. Flyvbjerg, Phys. Rev. E 47, 4037 (1993); Physica A 194, 298 (1993).

[14] J. Stavans, E. Domany, and D. Mukamel, Europhys. Lett. 15, 479 (1991).

[15] T. Aste, K.Y. Szeto, and W.Y. Tam, Phys. Rev. E 54, 5482 (1996).

[16] B. Levitan, E. Slepyan, O. Krichevshy, J. Stavans, and E. Domany, Phys. Rev. Lett. 73, 756 (1994); B. Levitan and E. Domany, Phys. Rev. E 54, 2766 (1996); Int. J. Mod. Phys. B B10, 3765 (1996).
[17] W.Y. Tam, K.M. Cheung, and K.Y. Szeto, Phys. Rev. E 57, 7354 (1998).

[18] B. Derrida, V. Hakim, and V. Pasquier, Phys. Rev. Lett. 75, 751 (1995); B. Derrida, V. Hakim, and R. Zeitak, ibid. 77, 2871 (1996); S.N. Majumdar, C. Sire, A.J. Bray, and S.J. Cornell, ibid. 77, 2867 (1996).

[19] W.Y. Tam, R. Zeitak, K.Y. Szeto, and J. Stavans, Phys. Rev. Lett. 78, 1588 (1997).

[20] W.Y. Tam, Physica A A288, 363 (2000).

[21] B. Dubetret, K.Y. Szeto, and W.Y. Tam, Europhys. Lett. 45, 143 (1999).

[22] B.P. Lee and A.D. Rutenberg, Phys. Rev. Lett. 79, 4842 (1997). Note that $\phi=1-\epsilon$, where $\epsilon$ is the minority volume fraction in that paper.

[23] W.Y. Tam, A.D. Rutenberg, B.P. Vollmayr-Lee, and K.Y. Szeto, Europhys. Lett. 51, 223 (2000).

[24] H.J. Ruskin and Y. Feng, J. Phys.: Condens. Matter 7, L553 (1995).

[25] D. Weaire and J. Kermode, Philos. Mag. B 48, 245 (1983); 47, L29 (1983); 48, 379 (1984); J. Kermode and D. Weaire, Comput. Phys. Commun. 60, 75 (1990).

[26] H. Aref and T. Herdtle, in Topological Fluid Mechanics, edited by H. Moffatt and A. Tsinober (Cambridge University Press, Cambridge, England, 1990), pp. 745-764; T. Herdtle and A. Aref, J. Fluid Mech. 241, 233 (1992).

[27] J. J. Chae, Ph. D. thesis, University of Arizona, 1995.

[28] J.J. Chae and M. Tabor, Phys. Rev. E 55, 598 (1996).

[29] M. Hennecke, Physica A 246, 519 (1997).

[30] B. Derrida, P.M.C. de Oliveira, and D. Stauffer, Physica A 224, 604 (1996); M. Hennecke, ibid. 252, 173 (1998). Note that in Fig. 4 we use Hennecke's data for $q>2(\phi<1 / 2)$.

[31] B. Levitan and E. Domany, Europhys. Lett. 38, 485 (1997).

[32] B. Yurke et al., Phys. Rev. E 56, 40 (1997).

[33] K. M. Cheung, K. Y. Szeto, and W. Y. Tam (unpublished). 